Case

\title{
Papillary Fibroelastoma of the Aortic Valve Visualized by 320-slice Computed Tomography: Report of a Case
}

\author{
Hajime Kin, MD, $\mathrm{PhD},{ }^{1}$ Junichi Koizumi, $\mathrm{MD}, \mathrm{PhD},{ }^{1}$ Kunihiro Yoshioka, $\mathrm{MD}, \mathrm{PhD},{ }^{2}$ and \\ Hitoshi Okabayashi, $\mathrm{MD}, \mathrm{PhD}^{1}$
}

\begin{abstract}
A 72-year-old man presented with a papillary fibroelastoma on the non-coronary cusp of the aortic valve. He was asymptomatic, with a history of hypertension and paroxysmal atrial fibrillation. Echocardiography revealed a mobile, round mass $(13 \times 15 \mathrm{~mm})$ on the non-coronary cusp of the aortic valve. Scanning with 320-slice multi-detector row computed tomography (MDCT) also revealed a mass on the non-coronary cusp of the aortic valve. The tumor was subsequently excised from the aortic valve. In this case, the MDCT images were extremely clear and provided useful information like that obtained with echocardiography.
\end{abstract}

Keywords: cardiac tumor, papillary fibroelastoma, cardiac surgery, computed tomography

\section{Introduction}

Cardiac papillary fibroelastoma $(\mathrm{CPF})$ is a rare, benign tumor, but is the third most common benign cardiac tumor after myxoma and lipoma. ${ }^{1,2)}$ We describe an asymptomatic man with the diagnosis of aortic valve papillary fibroelastoma that was incidentally found by echocardiography. This case also demonstrated the sensitivity of multi-detector row computed tomography (MDCT) for the diagnosis of rare cardiac tumors, especially when new techniques such as three-dimensional CT are employed to provide valuable information like that obtained with echocardiography.

${ }^{1}$ Department of Cardiovascular Surgery, Iwate Medical University Memorial Heart Center, Morioka, Iwate, Japan

${ }^{2}$ Department of Radiology, Iwate Medical University Memorial Heart Center, Morioka, Iwate, Japan

Received: July 9, 2010; Accepted: September 6, 2010

Corresponding author: Hajime Kin, MD, PhD. Department of Cardiovascular Surgery, Iwate Medical University Memorial Heart Center, 19-1 Uchimaru, Morioka, Iwate 020-8505, Japan

Email: h_kin@imu.ncvc.go.jp

(C)2011 The Editorial Committee of Annals of Thoracic and Cardiovascular Surgery. All rights reserved.

\section{Case Report}

A 72 year-old man was referred to our hospital because a cardiac tumor had been detected by routine echocardiographic examination. He was asymptomatic, with a history of hypertension and paroxysmal atrial fibrillation.

Transthoracic and transesophageal echocardiography (TTE and TEE) revealed a mobile, round mass $(13 \times$ $15 \mathrm{~mm}$ ) on the non-coronary cusp of the aortic valve without any aortic regurgitation or stenosis (Fig. 1). No left ventricular asynergy was seen. MDCT revealed a mass $(15 \mathrm{~mm} \times 10 \mathrm{~mm})$ attached to the ventricular surface of the non-coronary cusp of the aortic valve (Fig. 2). Coronary CT revealed no coronary artery stenosis. The diagnosis was papillary fibroelastoma of the aortic valve. We decided to remove the tumor because it was mobile and over $1 \mathrm{~cm}$ in size.

The operation was performed via midline sternotomy. After establishing cardiopulmonary bypass, the ascending aorta was cross-clamped. After opening the aorta, the tumor was found on the ventricular surface of the noncoronary cusp of the aortic valve (Fig. 3D). Macroscopically, the tumor resembled a sea anemone. The mass was excised sharply from the aortic valve. The size of the resected tumor was $15 \times 8 \mathrm{~mm}$, and the final pathologic diagnosis was papillary fibroelastoma. The postoperative 


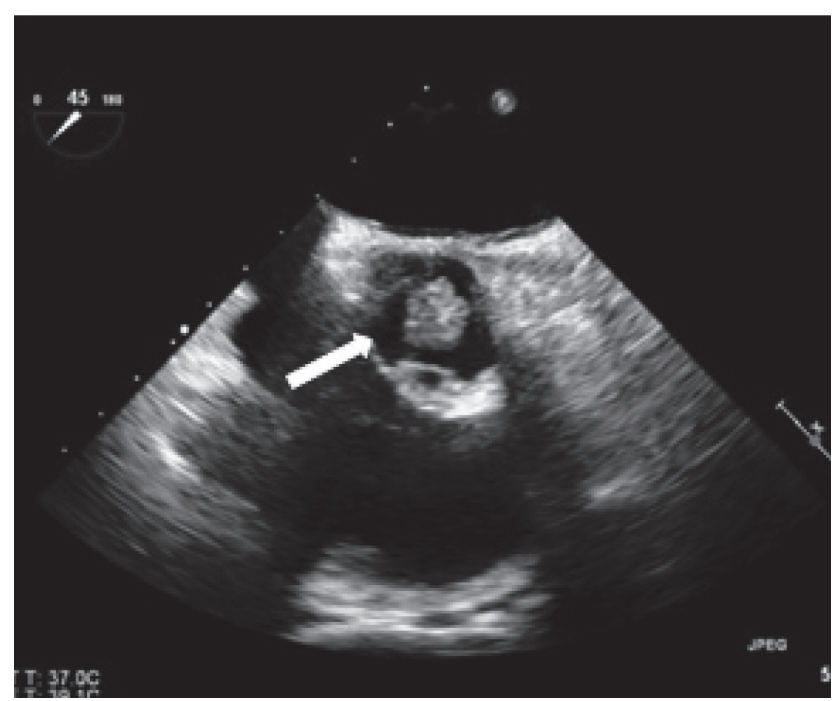

Fig. 1 Transesophageal echocardiography. Short-axis view of the aortic valve showing a non-coronary mass (arrow).

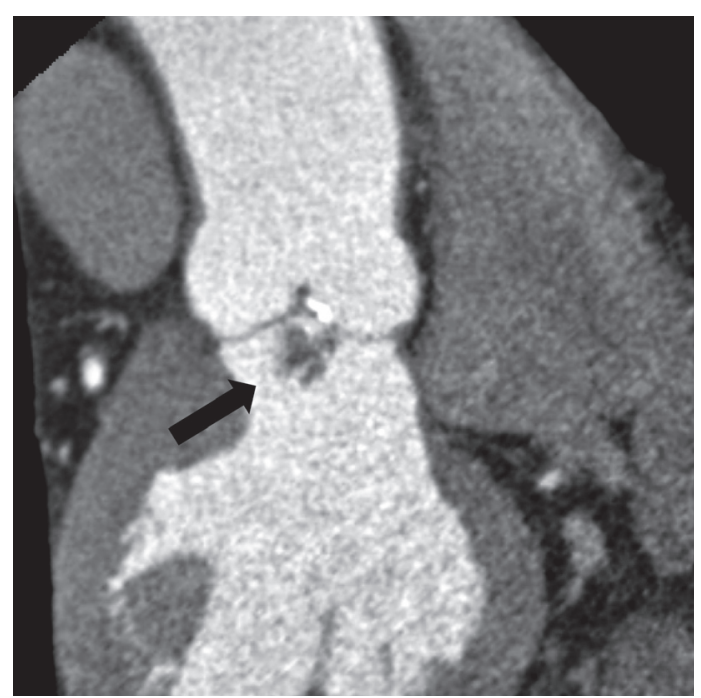

Fig. 2 Multislice spiral CT revealed a mass $(15 \mathrm{~mm} \times 10 \mathrm{~mm})$ attached to the ventricular surface of the non-coronary cusp of the aortic valve (arrow).
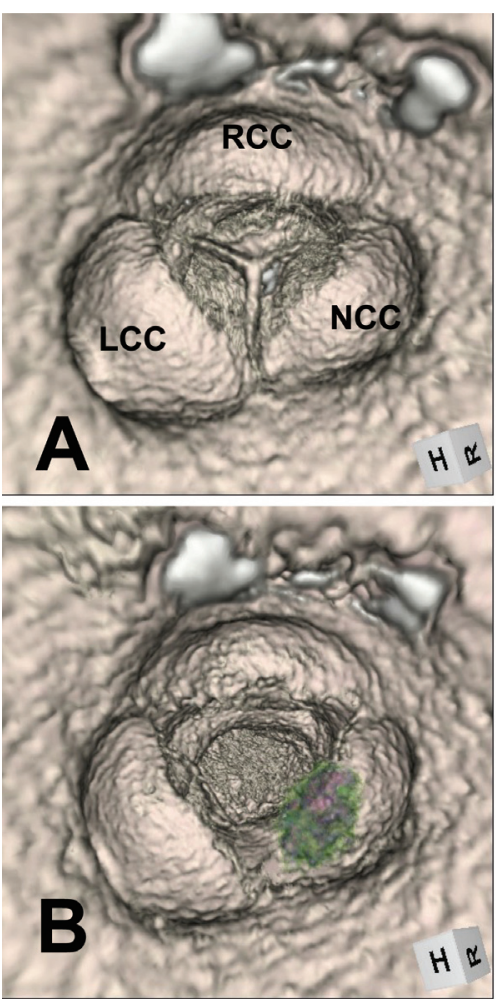
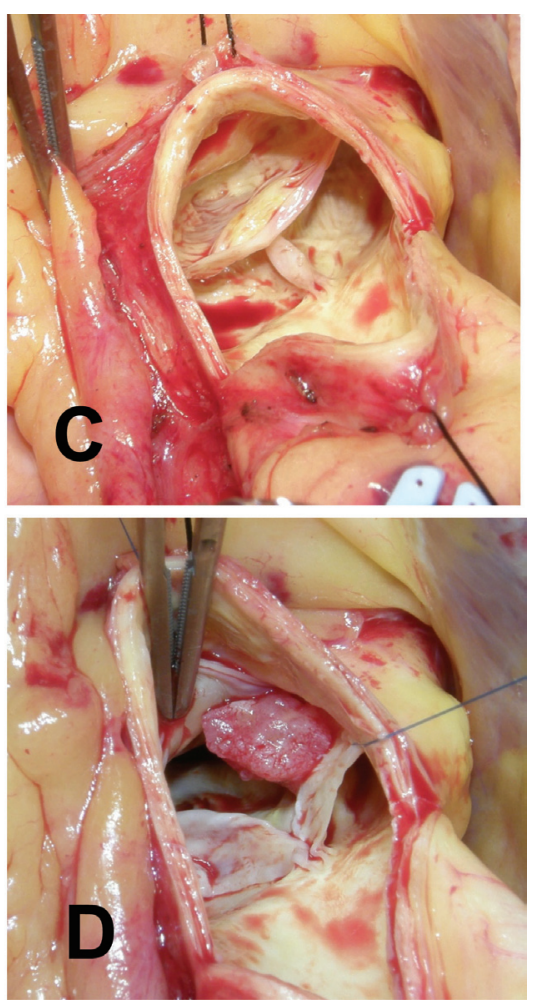

Fig. 3 A and B: Supra-annular three-dimensional image reconstructed using 320-slice multidetector row computed tomography. A mass (arrow) is attached to the non-coronary cusp of the aortic valve (A: diastolic phase, B: systolic phase).

C and D: At operation, a peduncular tumor $(15 \mathrm{~mm} \times 8 \mathrm{~mm})$ is located on the ventricular surface of the non-coronary cusp of the aortic valve (C: closed valve, D: opened valve). course was uneventful, and the patient was discharged 14 days after surgery.

\section{Discussion}

Primary intracardiac tumors are rare, with a prevalence ranging from 0.0017 to $0.28 \%$, and approximately
$75 \%$ of these tumors are benign. ${ }^{1,2)} \mathrm{CPF}$ accounts for less than $10 \%$ of cardiac tumors, but represents the most common valvular tumor and the second most common cardiac tumor following myxoma., ${ }^{3,4)}$

Needless to say, the widespread use of TTE as well as TEE has led to earlier diagnosis and treatment of intracardiac tumors. Two-dimensional echocardiography is the 
optimal noninvasive technique for imaging small masses $(<1 \mathrm{~cm})$ or masses arising from the heart valves, since it provides excellent morphological and functional information $^{1,5)}$ In addition, since Bootsvelt et al. ${ }^{6)}$ first reported the detection of CPE by MDCT, it has been used as a diagnostic tool along with echocardiography and magnetic resonance imaging. ${ }^{7-9)}$

A merit of MDCT is that, if the patient has suspected coronary artery disease, the presence of coronary disease can be evaluated noninvasively. Cardiac catheterization is associated with an added risk because the catheter may dislodge fragments of the tumor and cause embolism. ${ }^{10}$ Yoda et al. ${ }^{11)}$ also commented that catheter manipulation close to the aortic valve may cause embolization, when a cardiac mass is located on the valve. In contrast, MDCT is simple, safe and noninvasive procedure.

In about $30 \%$ of patients, CPF is an incidental finding at autopsy or during surgery for other cardiac conditions. ${ }^{10)} \mathrm{CPF}$ is characteristically a small, avascular, solitary tumor ${ }^{12)}$ with multiple spicules that resembles a sea anemone and is connected to the endocardium. Most lesions are less than $1 \mathrm{~cm}$ in diameter, but these tumors range in size from $\left.0.1-4 \mathrm{~cm} .{ }^{13}{ }^{14}\right)$ The size of the tumors reported by MDCT is around $9-10 \mathrm{~mm}^{7-9)}$ Valvular CPF arises almost exclusively in the mid-portion of the valve, without encroaching on the annulus or the free edge of the valve leaflet. In our patient, the tumor visualized by MDCT was typical. However, there have been recent reports of multiple tumors or rare anatomical locations, such as the left ventricular outflow tract, tricuspid valve, and right atrium. ${ }^{11,15,16)}$ At present, we have no idea how well small or multiple tumors will be detected by MDCT.

MDCT revealed that the tumor was located on the ventricular surface of the non-coronary cusp of the aortic valve (Figs. 2, 3A and 3B). The surgical findings (Fig. 3C and 3D) confirmed the accuracy of CT images. We emphasize that, in addition to TTE and TEE, preoperative evaluation by MDCT might help the cardiothoracic surgeon to identify aortic papillary fibroelastoma and is important for making management decisions regarding the performance of simple excision, valve repair, and valve replacement.

\section{References}

1) Burke A, Jeudy J Jr, Virmani R. Cardiac tumors: an update: cardiac tumor. Heart 2008; 94: 117-23.
2) Grande AM, Ragni T, Vigano M. Primary cardiac tumors. A clinical experience of 12 years. Tex Heart Inst J 1993; 20: 223-30.

3) Klarich KW, Entriquez-Sarano M, Gura GM, et al. Papillary fibroelastoma: echocardiographic characteristics for diagnosis and pathologic correction. J Am call Cardiol 1997; 30: 784-90.

4) Bossert T, Gummert JF, Battellini R, et al. Surgical experience with 77 primary cardiac tumors. Interact Cardiovasc Thorac Surg 2005; 4: 311-5.

5) Parthenakis F, Nyktari E, Patrianakos A, et al. Asymptomatic papillary fibroelastoma of the aortic valve in a young woman-a case report. Cardiovasc Ultrasound 2009; 7: 43 .

6) Bootsveld A, Puetz J, Grube E. Incidental finding of a papillary fibroelastoma on the aortic valve in 16 slice multi-detector row computed tomography. Heart 2004; 90: e35.

7) Czekajska-Chehab E, Tomaszewski A, Wojcik M, et al. Papillary fibroelastoma as an accidental finding in a multiple computed tomography scan of coronary arteries. Can J Cardiol 2006; 322: 1155-7.

8) Kondruweit M, Schmid M, Strecker T. Papillary fibroelastoma of the mitral valve: appearance in 64-slice spiral computed tomography, magnetic resonance imaging, and echocardiography. Eur Heart J 2008; 29: 831.

9) Lembcke A, Meyer R, Kivelita D, et al. Papillary fibroelastoma of the aortic valve. Appearance in 64-slice spiral computed tomography, magnetic resonance imaging, and echodcardiography. Circulation 2007; 115: e3-6.

10) Gowda RM, Khan IA, Nair CK, et al. cardiac papillary fibroelastoma: a comprehensive analysis of 725 cases. Am Heart J 2003; 146: 404-10.

11) Yoda M, Tanabe H, Kanou H, et al. Multiple papillary fibroelastoma in rare location of aortic valve and left ventricular outflow tract: a case report. J Heart Valve Dis 2009; 18:

12) Edwards FH, Hale D, Cohen A, et al. Primary cardiac valve tumors. Ann Thorac Surg 1991; 52: 1127-31.

13) Kasarski EJ, O'Connor W, Earle G. Embolic stroke from cardiac papillary fibroelastomas. Stroke 1988; 19: 1171-3.

14) Lee KS, Topol EJ, Stewart WJ. Atypical presentation of papillary fibroelastoma mimicking multiple vegetations in suspected bacterial endocarditis. Am Heart J 1993; 125: 1443-5.

15) Kobayashi Y, Saito S, Yamazaki K, et al. Multiple papillary fibroelastoma in left ventricle associated with obstructive hypertrophic cardiomyopathy. Interact Cardiovasc Thorac Surg 2009; 9: 921-2.

16) Maybury R, Mullenix P, Greengerg M, et al. Rare anatomical location of a papillary fibroelastoma. Ann Thorac Surg 2009; 88: 2034-5. 